\title{
JWA suppresses the invasion of human breast carcinoma cells by downregulating the expression of CXCR4
}

\author{
LINGYUN XU ${ }^{1,2^{*}}$, LIN CHENG $^{1 *}$, FANGLIANG YANG $^{1}$, BEI PEI $^{1}$, XIAOAN LIU $^{2}$, \\ JIANWEI ZHOU ${ }^{3}$, YULAN ZHU ${ }^{1}$ and SHUI WANG ${ }^{2}$
}

\begin{abstract}
${ }^{1}$ Department of Breast Surgery, The Affiliated Changzhou No. 2 People's Hospital of Nanjing Medical University, Changzhou, Jiangsu 213003; ${ }^{2}$ Department of Breast Surgery, The First Affiliated Hospital of Nanjing Medical University; ${ }^{3}$ Department of Molecular Cell Biology and Toxicology, Jiangsu Key Lab of Cancer Biomarkers, Prevention and Treatment, Cancer Center, School of Public Health, Nanjing Medical University, Nanjing, Jiangsu 210029, P.R. China
\end{abstract}

Received March 23, 2017; Accepted March 29, 2018

DOI: $10.3892 / \mathrm{mmr} .2018 .8866$

\begin{abstract}
Breast cancer is the second leading cause of cancer-associated mortality, and metastatic breast cancer is responsible for $90 \%$ of patient mortalities. Given that JWA represses the proliferation, invasion and metastasis of a number of other human tumor cells, including melanoma, esophageal, hepatocellular and gastric carcinomas, via mitogen-activated protein kinase or integrin signaling, the present study investigated the expression and function of JWA in human breast cancers. The results showed that the expression level of JWA was significantly reduced in human primary breast cancers when compared with the paired adjacent tissues. Downregulating JWA enhanced, while overexpressing JWA suppressed, the migration and invasion abilities of the two breast cancer cell lines, MDA-MB-468 and MDA-MB-231, without affecting their proliferations in vitro. In addition, JWA negatively regulated the surface expression of CXCR4 in the two cell lines via proteasome degradation, though not via transcriptional inhibition. Functionally, normalizing the disturbed expressions of CXCR4 largely reversed the inhibitory effect of JWA on cell invasion. These data demonstrated that JWA suppressed the migration/invasion of breast carcinoma cells by downregulating the expression of $\mathrm{CXCR} 4$, and
\end{abstract}

Correspondence to: Dr Yulan Zhu, Department of Breast Surgery, The Affiliated Changzhou No. 2 People's Hospital of Nanjing Medical University, 29 Xinglong Lane, Changzhou, Jiangsu 213003, P.R. China

E-mail: zhuyulanma@sina.com

Dr Shui Wang, Department of Breast Surgery, The First Affiliated Hospital of Nanjing Medical University, 300 Guangzhou Road, Nanjing, Jiangsu 210029, P.R. China

E-mail: ws0801@hotmail.com

*Contributed equally

Key words: JWA, breast cancer, cell invasion, C-X-C motif chemokine receptor type 4 suggested that JWA may harbor prognostic and therapeutic potential in patients with breast cancer.

\section{Introduction}

Breast cancer is the most prevalent cancer among females worldwide, with an incidence ranging from $0.02 \%$ in middle Africa to $1 \%$ in North America. Although the overall survival rate for patients has improved significantly in the past 30 years due to earlier diagnosis and better treatments, nowadays breast cancer still represents the second leading cause of cancer-associated mortality in women, mainly due to its invasion/metastasis to other organs including lungs and livers $(1,2)$. However, mechanisms responsible for the migration, invasion and metastasis of breast cancer cells remain poorly understood.

Chemokines belongs to a super-family of small, cytokine-like proteins that induce cytoskeletal rearrangement, adhesion to endothelial and directional migration by interacting with G-protein-coupled receptors (GPCRs) $(3,4)$. Among the 46 different human chemokines and 18 GPCRs identified so far, CXCL12 and its receptor CXCR4 play an important role in promoting the invasion and metastasis of breast cancers (4-6). It has been shown that breast cancer cells express aberrant high levels of CXCR4, and thereby metastasize preferentially to CXCL12-rich tissues such as lungs and bone marrows $(4,6)$. Accordingly, reducing the expression of CXCR4 or blocking CXCR4-CXCL12 interactions reduces the experimental invasion and metastasis of breast cancer cells (7-9). Thus, identifying molecules regulating the expression of CXCR4 on cancer cells may represent a potential target for the treatment of patients with breast cancer.

JWA, also known as ADP ribosylation factor like GTPase 6 interacting protein 5 (ARL6IP5), was initially cloned from human tracheal bronchial epithelial cells as an all-trans retinoic acid responsive and cytoskeleton-associated gene, and has been shown to be involved in cell oxidative stress, differentiation and apoptosis (10-15). In addition, as a microtubule-associated protein, JWA regulates the proliferation and migration of cancer cells via MAPK cascades, inhibits the invasion and 
metastasis of melanoma cells via integrin signaling, and suppresses the tumor angiogenesis via ILK signaling or Sp1-activated MMP-2 expressions (16-19). Therefore, the downregulated expression of JWA predicts poor prognosis in human melanoma, esophageal squamous cell carcinoma, hepatocellular carcinoma and gastric cancers (18-21). Nonetheless, the functions of JWA in breast cancers remain largely unknown.

In the present study, we found that the expression of JWA is significantly reduced in primary human breast tumors than the paired adjacent normal tissues. Despite the ineffectiveness in cellular proliferations, increasing the expression of JWA in breast cancer cell line MDA-MB-231 (with low endogenous JWA) greatly reduces the expression of CXCR4 and the in vitro cellular migration/invasion abilities, while downregulating its expressions in MDA-MB-468 cells (with high endogenous JWA) has the opposite effect. Moreover, preventing the down-/upregulation of CXCR4 induced by increased/decreased JWA expressions in breast carcinoma cells almost completely reverses the disturbed cellular invasions to control levels. These data indicate that JWA suppresses the migration/invasion of breast carcinoma cells via downregulating the expression of CXCR4. Our findings further strengthen the importance of JWA in tumor invasion and metastasis, and suggest that JWA may represent a potential anti-metastatic target for breast cancer patients.

\section{Materials and methods}

Breast cancer specimens. The tumor specimens and paired normal breast tissue specimens were obtained from patients undergoing breast surgery. None of the patients had received radiotherapy or chemotherapy prior to the surgery. Written informed consent was provided by each patient recruited and the present study was approved by the local human Ethics Committee of The Affiliated Changzhou No. 2 People's Hospital of Nanjing Medical University (Changzhou, Jiangsu, China).

Cell lines and culture. Breast carcinoma cells MDA-MB-231 and MDA-MB-468 were purchased from the Type Culture Collection of the Chinese Academy of Sciences (Shanghai, China). All the cells were cultured in DMEM medium supplemented with $10 \%$ of fetal bovine serum (FBS), $100 \mathrm{U} / \mathrm{ml}$ of penicillin and $100 \mu \mathrm{g} / \mathrm{ml}$ of streptomycin (Gibco; Thermo Fisher Scientific, Inc., Waltham, MA, USA) at $37^{\circ} \mathrm{C}$ in a humidified incubator with $5 \% \mathrm{CO}_{2}$.

Plasmids and transfection. The control Flag-vector and Flag-JWA plasmids were kindly provided by Professor Gang Li (University of British Columbia, Canada) as described previously (13). HA-tagged CXCR4 vector was obtained by subcloning the cDNA into the pCMV-HA-dsRed2 expression plasmid (GV316; Genechem, Shanghai, China). SiRNA specific for JWA (5'-CGAGCTATTTCCTTATCTC-3') was synthesized by Riobio (Guangzhou, China) as previously published (14). To specifically knockdown the expression of CXCR4, we subcloned the CXCR4-specific sequence (5'-TGCCTTACT ACATTGGGAT-3') into the pCMV-U6-GFP shRNA vector (GV248; Genechem). Cells were (co-) transfected with siRNA or plasmids with Lipofectamine 2000 following the protocols provided by the manufacturer (Invitrogen; Thermo Fisher, Inc.).

Reverse transcription-quantitative polymerase chain reaction $(R T-q P C R)$. RNA was isolated from cells with TRIzol (Takara Bio, Dalian, China) and reversely transcribed into cDNA using an oligo (dT) primer subsequently (Promega Corp., Madison, WI, USA). RT-qPCR was performed with SYBR Premix Ex Taq (Takara Bio) using an ABI 7900HT detection system (Thermo Fisher Scientific Inc.). Gene expression levels were normalized to the endogenous GAPDH in each sample.

Western blot analysis. Western blots were performed as previously described (12). Briefly, cells were lysed in keratin extraction buffer (1\% Triton-X 100, $0.02 \mathrm{mM}$ Tris, $0.6 \mathrm{M} \mathrm{KCl,}$ and $1 \mathrm{mM}$ PMSF, pH 7.0) and protein concentrations were determined by bicinchoninic acid (BCA) assays (Beyotime, Nantong, China). Proteins were separated in SDS-PAGE $12.5 \%$ gels and blotted onto PVDF membrane (Millipore). After incubation for $1 \mathrm{~h}$ in blocking buffer (Tris-buffered saline with $5 \%$ nonfat milk), the membrane was incubated with primary antibodies overnight at $4^{\circ} \mathrm{C}$, followed by a further incubation with HRP-coupled secondary antibodies at room temperature for $2 \mathrm{~h}$. Signals were visualized with an enhanced chemiluminescent kit (GE Healthcare, Chicago, IL, USA). The following antibodies were used: Mouse monoclonal anti-JWA (contract produced by AbMax, Beijing, China) and anti-GAPDH (6C5; Beyotime); rabbit polyclonal anti-Flag (Beyotime); rabbit monoclonal anti-CXCR4 (UMB2, Abcam); Rabbit monoclonal anti-AKT (C67E7) and anti-pAKT (D25E6; Cell Signaling Technology, Inc., Danvers, MA, USA ); and HRP-coupled polyclonal goat anti-mouse or rabbit IgG (Beyotime).

Transwell invasion assay. The 24-well Transwell chambers with a pore size of $8 \mathrm{~mm}$ (Corning, Tewksbury, MA, USA) were pre-coated with $50 \mathrm{ml} 100 \mathrm{mg} / \mathrm{ml}$ fibronectin (Sigma-Aldrich; Merck KGaA, Darmstadt, Germany). $10^{5}$ cells in $100 \mathrm{ml}$ serum-free medium were seeded into the upper chamber while $600 \mathrm{ml}$ medium with $10 \%$ serum was added into the lower chamber. After incubation at $37^{\circ} \mathrm{C}$ for $12 \mathrm{~h}$, cells in the upper chamber were carefully removed with a cotton swab and cells that had traversed to the reverse side of the membrane were fixed in methanol, stained with Giemsa, and imaged with a microscopy (IX70; Olympus Tokyo, Japan). Experiments were performed in triplicates, and five random fields of each well were recorded to count the cell numbers. In some experiments, cells were pretreated with a CXCR4 specific antagonist AMD3100 (octahydrochloride hydrate; Sigma-Aldrich; Merck $\mathrm{KGaA}$ ) at $100 \mathrm{nM}$ for $2 \mathrm{~h}$ before seeding.

Wound healing migration assays. Cells were seeded into 24-well plates and cultured till 80-90\% confluence. Cells were washed once with PBS, and then a scratch was gently introduced onto the cell monolayer with $200 \mathrm{ml}$ pipette tips. After washing with PBS twice, cells were grown in serum-free medium, and migrating cells were imaged with a microscopy (IX70; Olympus) at 36 or $48 \mathrm{~h}$. Experiments were performed in duplicates, and three random fields of each well were recorded. The rate of wound closure was 
A

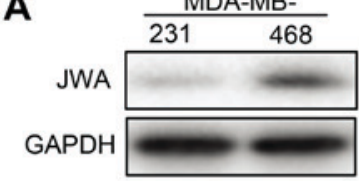

B

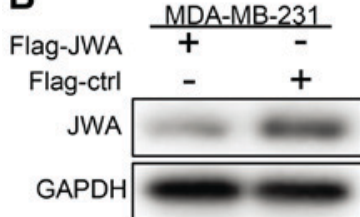

C

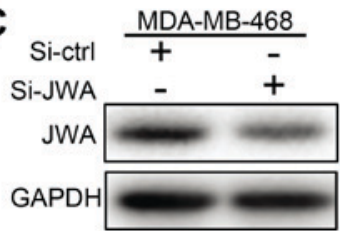

D

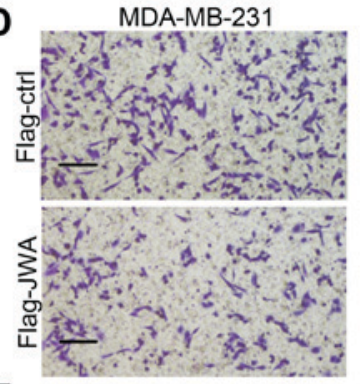

E

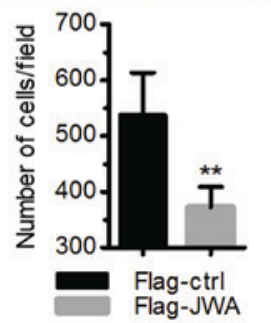

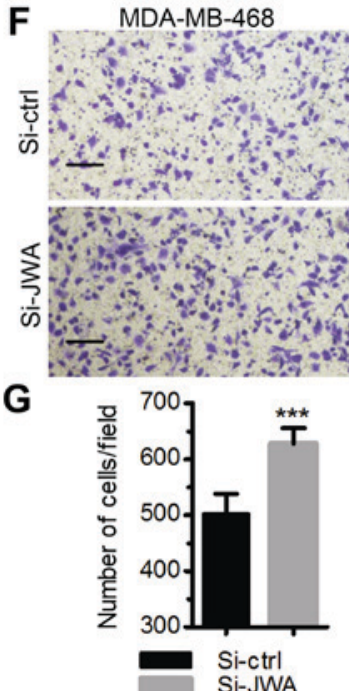

Figure 1. JWA negatively regulates the invasive ability of breast cancer cells. (A) MDA-MB-231 cells express lower levels of JWA than MDA-MB-468 cells as determined by western blot analysis. Overexpression of JWA inhibited the invasion ability of MDA-MB-231 cells. Cells were transfected with a control (Flag-ctrl) or CXCR4 expression plasmid (Flag-JWA) with Lipofectamine $2000^{\mathrm{TM}}$. Overexpression of JWA was confirmed by (B) western blotting and the (D) invasion ability was analyzed in (E) a Transwell assay following $48 \mathrm{~h}$. Knockdown of JWA increased the invasion ability of MDA-MB-468 cells. Cells were transfected with a control (Si-ctrl) or CXCR4-targeting siRNA (Si-JWA) with Lipofectamine $2000^{\mathrm{TM}}$. Downregulation of JWA was confirmed by (C) western blotting and the (F) invasion abilities were compared in a (G) Transwell assay $48 \mathrm{~h}$ post-transfection. Cells that had traversed to the reverse side of the membrane were fixed, stained and imaged (magnification, $\mathrm{x} 400$; scale bars $=100 \mu \mathrm{m}$ ). Experiments were performed in triplicate, and five random fields in each well were recorded to count the cell numbers. Results were expressed as the mean number \pm standard error of the mean of three independent experiments. ${ }^{* *} \mathrm{P}<0.01$ and ${ }^{* * *} \mathrm{P}<0.001$, vs. the Flag-/Si-control. CXCR4, C-X-C motif chemokine receptor type 4; ctrl, control; Si/siRNA, small interfering RNA.

calculated on the basis of the average distance between the two wound edges.

Proliferation assay. Cells were plated at a density of 5,000 cells/well in triplicates in 96-well plates. Cellular viabilities were determined by Cell Counting kit-8 (CCK-8; Beyotime) at indicated times according to the manufacturer's instructions. Proliferation index was calculated as the ratio of OD value at the indicated time/OD value of the input cells.

Statistical analysis. The differences among different groups were determined by the parametric unpaired Student's t-test, and values at $\mathrm{P}<0.05$ were considered significant.

\section{Results}

JWA negatively regulates the invasion/migration abilities of breast cancer cells. We first analyzed the expression levels of JWA in two breast cell lines, MDA-MB-231 and MDA-MB-468, with differential migratory abilities. Our data clearly showed that the endogenous protein level of JWA is significantly lower in MDA-MB-231 than that in MDA-MB-468 cells (Fig. 1A). As the endogenous levels of JWA seem to inversely correlate with cellular invasion/migration abilities (22), we subsequently downregulated/upregulated the protein levels of JWA in MDA-MB-468/MDA-MB-231 cells and determined its impact on cellular invasion/migration capabilities in vitro. Ectopically high expression of JWA in MDA-MB-231 cells remarkably repressed their invasion abilities in a Transwell assay (Fig. 1B, $\mathrm{D}$ and $\mathrm{E})$. By contrast, knockdown of endogenous JWA by siRNA significantly increased the number of MDA-MB-468 cells that had traversed the membrane (Fig. 1C, F and G). Similar results were obtained in would healing migration assays (Fig. 2A and B). Notably, this observed effect of JWA on cellular migration/invasion cannot be attributed to the disturbed cellular proliferations as manipulation of JWA levels has no impact on the proliferations of MDA-MB-231 or MDA-MB-468 cells (Fig. 2C). Moreover, side-by-side comparisons between the primary tumor and paired adjacent normal tissues revealed significantly lower levels of JWA in tumor tissues in all six breast cancer patients (Fig. 3A). Together, these data indicate that JWA negatively regulates the invasion/migration capabilities of breast carcinoma cells.

JWA downregulates the expression of CXCR4 in breast carcinoma cells. In line with previous reports (4), lower levels of CXCR4 were observed in breast tumors than the paired normal tissues (Fig. 3A). Given that CXCR4 promotes the in vitro migratory as well as the in vivo metastatic abilities of breast cancer cells through the PI3K/AKT signaling pathway $(4,6,23,24)$, the inversed correlation of JWA and CXCR4 levels in breast cancer tissues prompted us to investigate whether JWA regulates the expression of CXCR4 and/or phosphorylated AKT (p-AKT). As shown in Fig. 3B, overexpression of JWA in MDA-MB-231 cells represses the expression of CXCR4 and p-AKT, while knockdown of JWA in MDA-MB-468 cells has the opposite effect (Fig. 3B). We next down-/upregulated the expression of CXCR4 in MDA-MB-231/468 cells with shRNA/overexpression plasmids, respectively, to determine whether it affects JWA levels as well. As expected, levels of p-AKT were positively correlated with CXCR4 in both cell lines. However, comparable expressions of JWA were observed in control and MDA-MB-468/231 cells with up-/downregulated CXCR4 (Fig. 3C). We thus concluded that JWA represses the expression of CXCR4 and AKT signaling pathway in human breast cancer cells. 
A

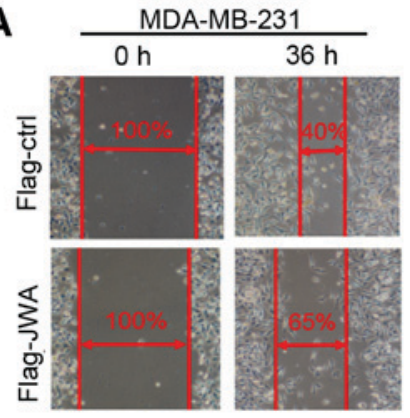

B

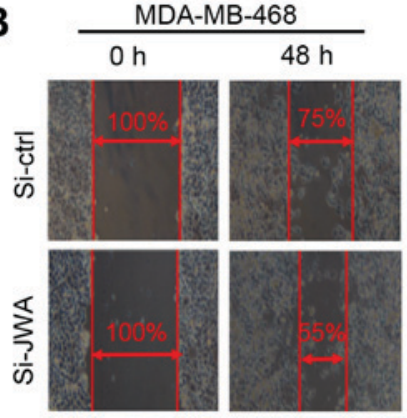

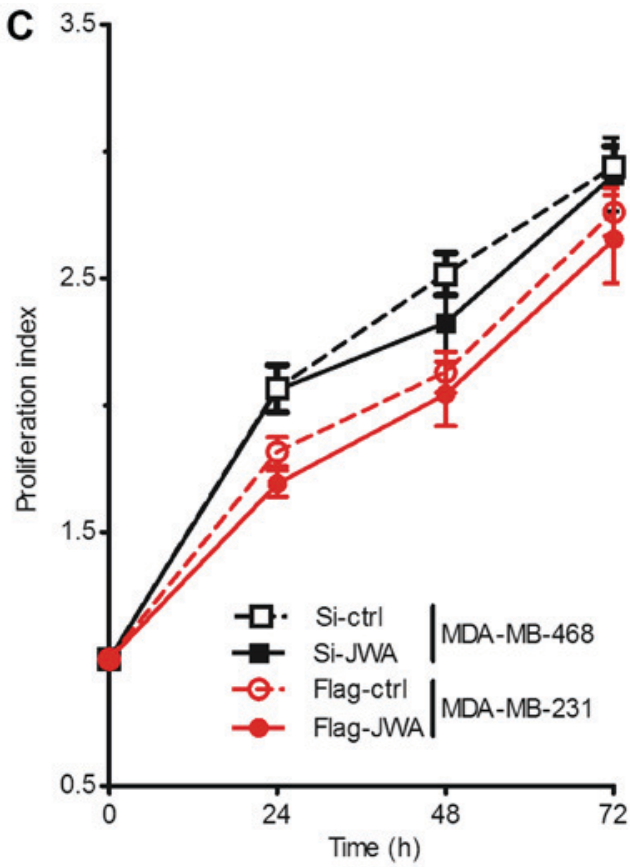

Figure 2. JWA negatively regulated the migratory ability of breast cancer cells without affecting their proliferation. (A) Overexpression of JWA inhibited the migration of MDA-MB-231 cells. Cells were transfected with a control (Flag-ctrl) or JWA-expressing plasmid (Flag-JWA) with Lipofectamine 2000 ${ }^{\mathrm{TM}}$. (B) Knockdown of JWA enhanced the migration of MDA-MB-468 cells. MDA-MB-468 cells were transfected with a control (Si-ctrl) or JWA-targeting siRNA (Si-JWA) with Lipofectamine $2000^{\mathrm{TM}}$. The cellular migratory abilities were determined by a wound healing assay 36 or $48 \mathrm{~h}$ post-transfections (magnification, $\mathrm{x} 400$ ). (C) JWA had no effect on the proliferation of MDA-MB-231 or MDA-MB-468 cells. Following $48 \mathrm{~h}$ post-transfection, 5,000 cells/well were plated in triplicate in 96-well plates and Cell Counting kit- 8 solution was added to the wells at the indicated times. Proliferation index was calculated as the ratio of the OD value at the indicated time/OD value of the input cells. Results were expressed as the mean \pm standard error mean of three independent experiments ctrl, control; Si/siRNA, small interfering RNA; OD, optical density.

A

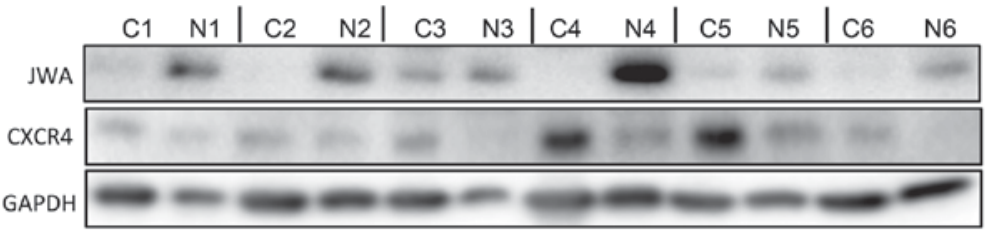

B

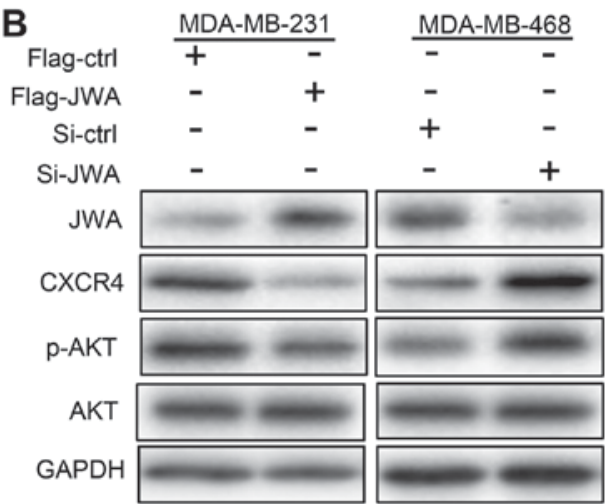

C

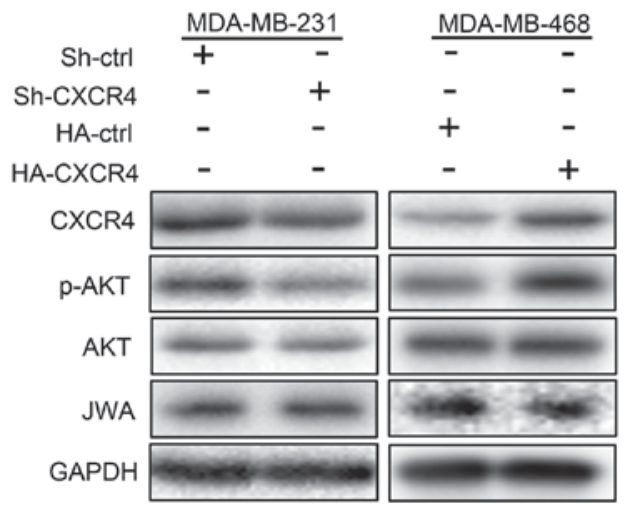

Figure 3. JWA suppresses the expression of CXCR4 and the activation of AKT in breast cancer cells. (A) Expression of JWA and CXCR4 in paired cancer (indicated by $\mathrm{C}$ ) and adjacent normal (indicated by $\mathrm{N}$ ) tissues taken from 6 breast cancer patients. (B) Overexpression and downregulation of JWA in MDA-MB-231 (left panel) and MDA-MB-468 (right panel) cells, respectively, resulted in decreased and increased expressions of CXCR4 and p-AKT, respectively. (C) Manipulations of CXCR4 levels in MDA-MB-231 and MDA-MB-468 cells do not affect the expression of JWA. MDA-MB-231 cells were transfected with a control (Sh-ctrl) or CXCR4-targeting vector to knockdown endogenous CXCR4 expressions. By contrast, MDA-MB-468 cells were transfected with a control (HA-ctrl) or CXCR4-expression (HA-CXCR4) plasmid to overexpress CXCR4. Levels of each protein were detected by western blotting $48 \mathrm{~h}$ post-transfection. CXCR4, C-X-C motif chemokine receptor type 4; ctrl, control; Si, small interfering RNA; Sh, small hairpin RNA; p-, phosphorylated; AKT, protein kinase B.

JWA suppresses the invasion abilities of breast cancer cells lines via downregulating the expression of CXCR4. Data presented in Figs. 1-3 implied that JWA may inhibit the invasion of breast cancer cells by regulating the expression of CXCR4. We thus tried to normalize the disturbed expressions of CXCR4 in MDA-MB-231/468 cells caused 
A

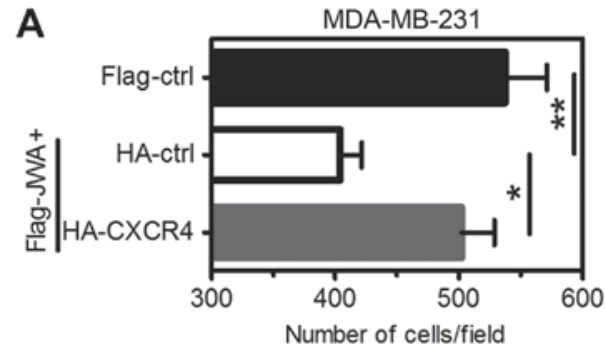

B

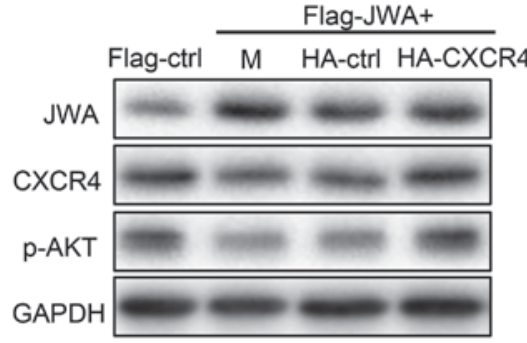

C

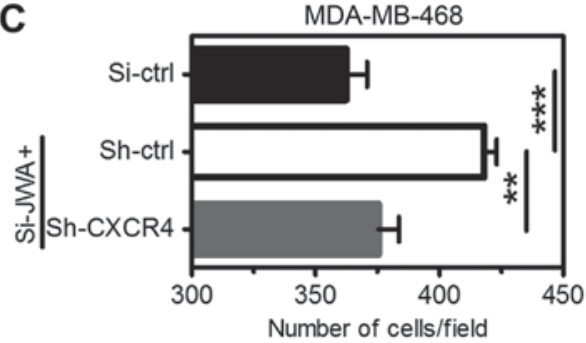

D

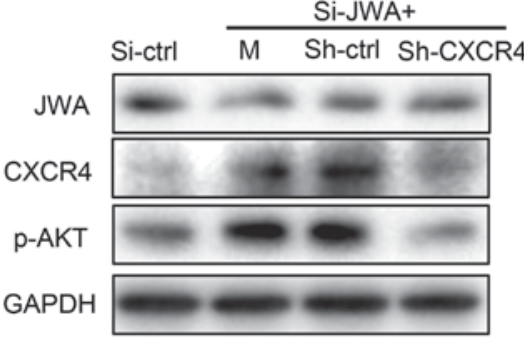

Figure 4. JWA inhibited the invasion abilities of breast cancer cells via CXCR4. (A) Preventing the downregulation of CXCR4 induced by the overexpressed JWA reversed the reduced cellular invasion ability of MDA-MB-231 cells. (B) Cells were co-transfected with a JWA-expressing vector (Flag-JWA) in the absence (indicated by $\mathrm{M}$ ) or presence of a CXCR4-expressing (HA-CXCR4), or a control (HA-ctrl) construct by Lipofectamine $2000^{\mathrm{TM}}$. (C) Downregulation of CXCR4 inhibited the augmented cellular invasion of MDA-MB-468 cells induced by the decreased levels of endogenous JWA. (D) MDA-MB-468 cells were co-transfected with a JWA-targeting siRNA (Si-JWA) in the absence (indicated by M) or presence of a CXCR4-targeting (Sh-CXCR4), or control (Sh-ctrl) shRNA-containing plasmids using Lipofectamine $2000^{\mathrm{TM}}$. Cellular invasive capabilities were determined in Transwell assays (A and C) and levels of each protein were analyzed by western blotting (B and D) $48 \mathrm{~h}$ post-transfection. Cells that had traversed to the reverse side of the membrane were fixed, stained and imaged. Experiments were performed in triplicate, and 5 random fields in each well were recorded to count the cell numbers. The results were expressed as the mean number \pm standard error mean of three independent experiments. ${ }^{~} \mathrm{P}<0.05,{ }^{* *} \mathrm{P}<0.01$ and ${ }^{* * *} \mathrm{P}<0.001$, as indicated. CXCR4, C-X-C motif chemokine receptor type 4; ctrl, control; Si, small interfering RNA; Sh, small hairpin RNA; p-, phosphorylated; AKT, protein kinase B.

by the overexpression/knockdown of JWA before measuring cellular invasion abilities. As shown in Fig. 4A and B, despite the high expression of JWA, MDA-MB-231 cells cotransfected both with JWA and CXCR4 expression plasmids displayed comparable levels of CXCR4 and p-AKT as control cells. Importantly, upregulation of CXCR4 almost
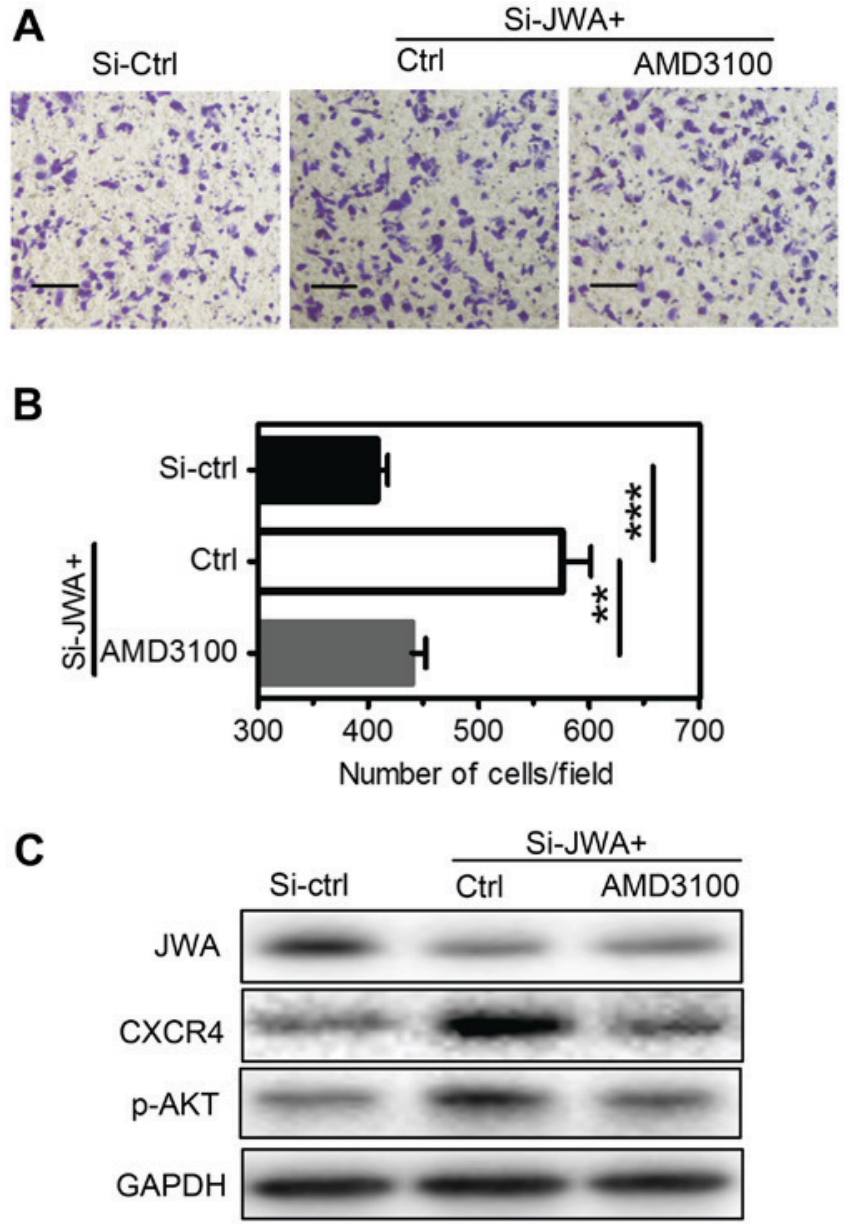

Figure 5. JWA inhibits the invasive abilities of breast cancer cells via the CXCR4-mediated signaling pathway. (A) MDA-MB-468 cells were transfected with a control (Si-ctrl) or JWA-targeting siRNA (Si-JWA) with Lipofectamine $2000^{\mathrm{TM}}$. Following $48 \mathrm{~h}$, cells were treated with a vehicle control (Ctrl) or AMD3100 (a CXCR4 specific antagonist) at $100 \mathrm{nM}$ for $2 \mathrm{~h}$. Cellular invasion abilities were then determined by (B) Transwell assays and protein levels were measured by $(C)$ western blot analysis. Cells that had traversed to the reverse side of the membrane were fixed, stained and imaged (magnification, $\mathrm{x} 400$; scale bars $=100 \mu \mathrm{m}$ ). Experiments were performed in triplicate, and 5 random fields of each well were recorded to count the cell numbers. The results were expressed as the mean number \pm standard error mean of three independent experiments. ${ }^{* * *} \mathrm{P}<0.01$ and ${ }^{* * * *} \mathrm{P}<0.001$, as indicated. CXCR4, C-X-C motif chemokine receptor type 4; ctrl, control; $\mathrm{Si}$, small interfering RNA; p-, phosphorylated; AKT, protein kinase B.

completely abrogated the inhibitory effect of overexpressed JWA on cellular invasion abilities in MDA-MB-231 cells (Fig. 4A). Likewise, preventing the upregulation of CXCR4 and p-AKT by transfecting MDA-MB-468 cells with a CXCR4-targeting shRNA vector reversed the enhanced cellular invasions conferred by downregulated JWA (Fig. 4C and D). Moreover, blocking CXCR4 signaling by treating JWA-downregulated MDA-MB-468 cells with AMD3100 (a CXCR4 specific antagonist) greatly reduced their transmigrations across the membrane in Transwell assays (Fig. 5). Therefore, cellular invasion capabilities are closely correlated to the expression levels of CXCR4/p-AKT, but not JWA, in both cell lines.

Collectively, these data indicate that JWA negatively regulates the invasion/migration abilities of breast cancer cells by inhibiting the expression of CXCR4. 
JWA inhibits the expression of CXCR4 by promoting protein degradations. To explore how JWA regulates the expression of CXCR4 in breast cancer cells, we first measured the mRNA levels of CXCR4 in MDA-MB-231/468 cells upon JWA upor downregulation, respectively. Interestingly, in contrast to the greatly reduced CXCR4 protein levels, comparable mRNA levels of CXCR4 were detected in MDA-MB-231 cells with overexpressed JWA (Figs. 4B and 6A). Similar results were obtained in MDA-MB-468 cells after knockdown of JWA by siRNA (data not shown). These data clearly demonstrated that JWA has no effect on the transcription of CXCR4 in breast cancer cells. Given that JWA is capable of suppressing protein expressions via ubiquitin-proteasome-dependent mechanism $(14,18)$, we subsequently treated control or JWA-overexpressing MDA-MB-231 cells with Cycloheximide (CHX, a protein synthesis inhibitor) or Bortezomib (PS341, a proteasome inhibitor). We found that overexpressed JWA gradually but significantly reduced the protein levels of CXCR4 in cells after Cycloheximide treatment (Fig. 6B), which was largely reversed by blocking the function of proteasomes (Fig. 6C). These data indicate that JWA promotes the proteasome degradation of CXCR4 and thereby negatively regulates its expression in breast cancer cells.

\section{Discussion}

In the present study, we investigated the expression and function of JWA in breast cancer cells as well as the mechanisms behind. Our data demonstrated a significantly reduced expression of JWA in primary breast cancers than paired adjacent normal tissues. Moreover, downregulating JWA increases while overexpressing JWA inhibits the in vitro cellular migration and invasion abilities of two breast cancer cell lines via modulating the proteasome degradation of CXCR4. Our study thus not only confirms the well-accepted negative roles of JWA in cancer invasion and metastasis, but also extends these studies by uncovering that JWA exerts this function via a previously unidentified mechanism, regulating the surface expression of chemokine receptor CXCR4 (16-21). JWA thus may represent a potential target for the treatment of breast cancer patients.

Numerous studies have indicated that JWA is a tumor suppressor gene as its downregulation promotes the survival, migration, invasion, metastasis and angiogenesis of a variety of human cancer cells (14,16-18,20,21,25-27). Regarding breast cancers, we have previously shown that JWA enhances $\mathrm{As}_{2} \mathrm{O}_{3}$-induced apoptosis of MCF-7 cells $(12,13)$. Moreover, Chen et al (28) recently demonstrated that human primary breast cancer tissues express significantly lower levels of JWA, and its knockdown promotes the proliferation, migration and invasion of MDA-MB-231 cells in vitro. By using two different breast cancer cell lines, we confirmed Chen's findings except the negative role of JWA in cellular proliferations (Figs. 1-4). This discrepancy may be attributed to different approaches used as Chen et al (28) downregulated while we upregulated JWA in MDA-MB-231 cells expressing low levels of endogenous JWA (Figs. 1 and 2). It is possible that cellular survival and proliferations will only be affected when the expression level of JWA, a microtubule- and cytoskeleton-associated protein,
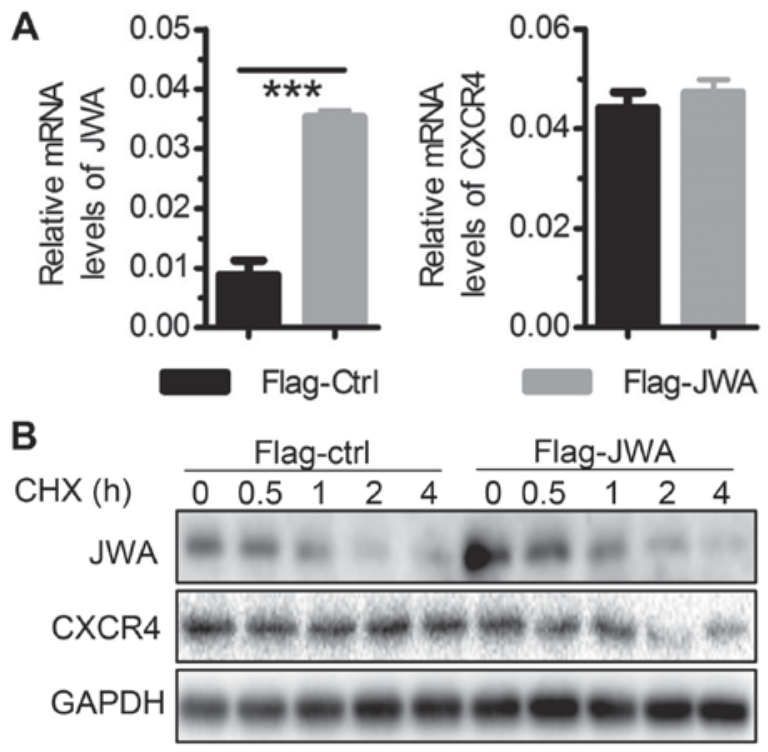

C

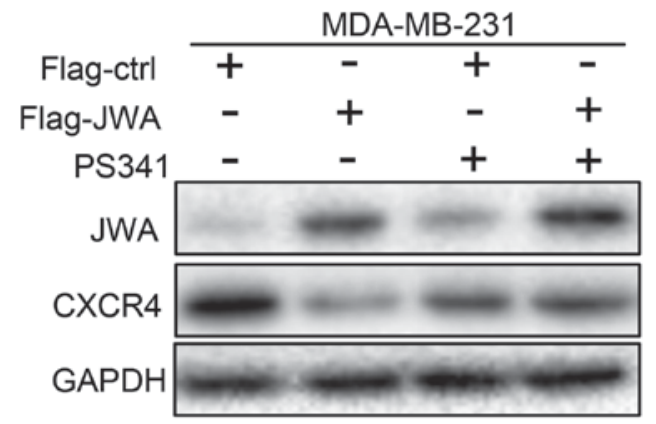

Figure 6. JWA downregulated the expression of CXCR4 by promoting protein degradation. MDA-MB-231 cells were transfected with a control (Flag-ctrl) or JWA-expression (Flag-JWA) vector by Lipofectamine $2000^{\mathrm{TM}}$. (A) Overexpressed JWA had no effect on the mRNA levels of CXCR4. The mRNA levels of JWA (left panel) and CXCR4 (right panel) were analyzed by reverse transcription-quantitative polymerase chain reaction $48 \mathrm{~h}$ post-transfection. Levels were normalized to the endogenous expression of the house-keeping gene GAPDH. Overexpressed JWA promoted the protein degradation of CXCR4. Control or JWA-overexpressing cells, $48 \mathrm{~h}$ post-transfection, were treated with (B) a protein synthesis inhibitor (CHX; $50 \mu \mathrm{g} / \mathrm{ml}$ ) for the indicated time or (C) a proteasome inhibitor Bortezomib (PS341; $50 \mu \mathrm{M}$ ) for $6 \mathrm{~h}$, followed by protein extraction and western blot analysis. ${ }^{* * *} \mathrm{P}<0.001$, as indicated. CXCR4, C-X-C motif chemokine receptor type 4; ctrl, control; CHX, cycloheximide.

is below a certain threshold. In this scenario, comparable proliferations between control and JWA-downregulated MDA-MB-468 cells could be explained by that the level of JWA in shRNA-treated cells was still above this threshold due to the high endogenous expressions of JWA (Figs. 1 and 2). Nevertheless, these data indicate that low expressions of JWA represent a bad prognostic maker for human breast cancers, in addition to previously reported melanoma, esophageal squamous, hepatocellular and gastric cancers (18-21).

Despite the high efficacy of current mainstream strategies including surgical resection and adjuvant therapies for well-confined primary breast tumors, metastatic breast cancer remains largely incurable and is responsible for $90 \%$ of the patient deaths (6). Given the role of 'chemokine-receptor' in metastasis and the most common expression of CXCR4 in human solid tumors, recent studies have drawn much attention to its role in tumor metastasis (9). 
It has been shown that CXCR4 is highly expressed in human breast cancer tissues and cell lines. Moreover, the expression levels of CXCR4 are closely correlated with the lymph node metastasis $(4,6,24)$, and antagonizing or silencing of CXCR4 blocks the metastasis of breast cancer cells (7-9). We thus investigated the potential crosstalks between JWA and CXCR4 by first measuring the expressions of CXCR4 and JWA in paired breast tumors and adjacent normal tissues side-by-side. In this western-blot assay, expressions of JWA $(\sim 21 \mathrm{Kd})$ and CXCR4 $(\sim 40 \mathrm{Kd})$ were detected on the same membrane, while GAPDH ( 36 Kd) was blotted on another membrane/gel loaded with the same amount of proteins as the protein size for CXCR4 and GAPDH are too close to be distinguished in one membrane. Moreover, a negative control (slot without proteins) was not included because of the limited loading slots. Nonetheless, our data showed that levels of CXCR4 are inversely-correlated with that of JWA in all human primary samples (Fig. 3A). Furthermore, we found that JWA is capable of repressing the migration and invasion of breast cancer cells through promoting the protein-degradation of CXCR4 (Figs. 3B-6). Together, these data indicated that JWA represents a novel negative regulator of CXCR4 in breast cancers, albeit that the detailed mechanisms behind merit further investigations. Additionally, given the broad expression of CXCR4 in human solid tumors (9), it is worth investigating whether findings described here can be extrapolated to other tumor types.

In conclusion, we have demonstrated that the expression of JWA is significantly reduced in primary human breast tumors, and more importantly, that JWA represses the migration and invasion of breast tumor cell lines via promoting the protein degradation of CXCR4. These findings are relevant as they suggest that JWA may represent not only a prognostic maker but also an anti-metastatic therapeutic target for patients with breast cancers.

\section{Acknowledgements}

The authors would like to thank Dr Jin Xu, Dr Qiang Wang and Dr Yuling Huang (School of Public Health, Nanjing Medical University, Jiangsu, China) for their valuable comments, and expertise in performing experiments and preparing figures.

\section{Funding}

The present study was financially supported in part by the National Natural Science Foundation of China (grant no. 81502294) and the Applied Basic Research Project of Changzhou (grant no. CZ20160035).

\section{Availability of data and materials}

All data generated or analyzed during this study are included in this published article.

\section{Authors' contributions}

LX and LC conceived and designed the study, performed the experiments, analyzed the data and drafted the manuscript. FY and BP were involved in recruiting patients and collecting patients' samples. XL performed the experiments, and JZ was involved in the study design and manuscript preparation. YZ and SW conceived and designed the study, analyzed the data and drafted the manuscript.

\section{Ethics approval and consent to participate}

Written informed consent was provided by each patient recruited and the present study was approved by the local Human Ethics Committee of The Affiliated Changzhou No. 2 People's Hospital of Nanjing Medical University.

\section{Consent for publication}

Written informed consent was provided by each patient recruited.

\section{Competing interests}

The authors declare that they have no competing interests.

\section{References}

1. Geiger TR and Peeper DS: Metastass mechanisms. Biochim Biophys Acta 1796: 293-308, 2009.

2. Miller KD, Siegel RL, Lin CC, Mariotto AB, Kramer JL, Rowland JH, Stein KD, Alteri R and Jemal A: Cancer treatment and survivorship statistics, 2016. CA Cancer J Clin 66: 271-289, 2016.

3. Cabioglu N, Gong Y, Islam R, Broglio KR, Sneige N, Sahin A Gonzalez-Angulo AM, Morandi P, Bucana C, Hortobagyi GN and Cristofanilli M: Expression of growth factor and chemokine receptors: New insights in the biology of inflammatory breast cancer. Ann Oncol 18: 1021-1029, 2007.

4. Wu W, Qian L, Chen X and Ding B: Prognostic significance of CXCL12, CXCR4, and CXCR7 in patients with breast cancer. Int J Clin Exp Pathol 8: 13217-13224, 2015.

5. Fernandis AZ, Prasad A, Band H, Klösel R and Ganju RK: Regulation of CXCR4-mediated chemotaxis and chemoinvasion of breast cancer cells. Oncogene 23: 157-167, 2004.

6. Mukherjee D, Lu H, Yu L, He C, Lahiri SK, Li T and Zhao J: Kruppel-like factor 8 activates the transcription of C-X-C cytokine receptor type 4 to promote breast cancer cell invasion, transendothelial migration and metastasis. Oncotarget 7: 23552-23568, 2016.

7. Huang EH, Singh B, Cristofanilli M, Gelovani J, Wei C, Vincent L, Cook KR and Lucci A: A CXCR4 antagonist CTCE-9908 inhibits primary tumor growth and metastasis of breast cancer. J Surg Res 155: 231-236, 2009.

8. Liang Z, Yoon Y, Votaw J, Goodman MM, Williams L and Shim H: Silencing of CXCR4 blocks breast cancer metastasis. Cancer Res 65: 967-971, 2005.

9. Katkoori VR, Basson MD, Bond VC, Manne U and Bumpers HL: Nef-M1, a peptide antagonist of CXCR4, inhibits tumor angiogenesis and epithelialtomesenchymal transition in colon and breast cancers. Oncotarget 6: 27763-27777, 2015.

10. Chen R, Qiu W, Liu Z, Cao X, Zhu T, Li A, Wei Q and Zhou J: Identification of JWA as a novel functional gene responsive to environmental oxidative stress induced by benzo[a]pyrene and hydrogen peroxide. Free Radic Biol Med 42: 1704-1714, 2007.

11. Huang S, Shen Q, Mao WG, Li AP, Ye J, Liu QZ, Zou CP and Zhou JW: JWA, a novel signaling molecule, involved in the induction of differentiation of human myeloid leukemia cells. Biochem Biophys Res Commun 341: 440-450, 2006.

12. Zhou J, Ye J, Zhao X, Li A and Zhou J: JWA is required for arsenic trioxide induced apoptosis in HeLa and MCF-7 cells via reactive oxygen species and mitochondria linked signal pathway. Toxicol Appl Pharmacol 230: 33-40, 2008.

13. Shen L, Xu W, Li A, Ye J and Zhou J: JWA enhances $\mathrm{As}_{2} \mathrm{O}_{3}$-induced tubulin polymerization and apoptosis via p38 in HeLa and MCF-7 cells. Apoptosis 16: 1177-1193, 2011. 
14. Xu W, Chen Q, Wang Q, Sun Y, Wang S, Li A, Xu S, Røe OD, Wang $\mathrm{M}$, Zhang $\mathrm{R}$, et al: JWA reverses cisplatin resistance via the CK2-XRCC1 pathway in human gastric cancer cells. Cell Death Dis 5: e1551, 2014.

15. Wei B, Han Q, Xu L, Zhang X, Zhu J, Wan L, Jin Y, Qian Z, Wu J, Gao Y, et al: Effects of JWA, XRCC1 and BRCA1 mRNA expression on molecular staging for personalized therapy in patients with advanced esophageal squamous cell carcinoma. BMC Cancer 15: 331, 2015.

16. Chen H, Bai J, Ye J, Liu Z, Chen R, Mao W, Li A and Zhou J: JWA as a functional molecule to regulate cancer cells migration via MAPK cascades and F-actin cytoskeleton. Cell Signal 19: $1315-1327,2007$

17. Bai J, Zhang J, Wu J, Shen L, Zeng J, Ding J, Wu Y, Gong Z, $\mathrm{Li} \mathrm{A}, \mathrm{Xu} \mathrm{S}$, et al: JWA regulates melanoma metastasis by integrin alphaVbeta3 signaling. Oncogene 29: 1227-1237, 2010.

18. Chen Y, Huang Y, Huang Y, Xia X, Zhang J, Zhou Y, Tan Y, He S, Qiang F, Li A, et al: JWA suppresses tumor angiogenesis via Spl-activated matrix metalloproteinase-2 and its prognostic significance in human gastric cancer. Carcinogenesis 35 442-451, 2014

19. Lu J, Tang Y, Farshidpour M, Cheng Y, Zhang G, Jafarnejad SM, Yip A, Martinka M, Dong Z,Zhou J, et al: JWA inhibits melanoma angiogenesis by suppressing ILK signaling and is an independent prognostic biomarker for melanoma. Carcinogenesis 34: 2778-2788, 2013.

20. Zhou J, Ge Z, Tan Y, Jiang G, Feng J, Wang H and Shi G: Downregulation of JWA expression in human esophageal squamous cell carcinoma and its clinical significance. Oncol Res 20: $157-162,2012$

21. Wu X, Chen H, Gao Q, Bai J, Wang X, Zhou J, Qiu S, Xu Y, Shi Y, Wang X, et al: Downregulation of JWA promotes tumor invasion and predicts poor prognosis in human hepatocellular carcinoma. Mol Carcinog 53: 325-336, 2014

22. Lim RC, Price JT and Wilce JA: Context-dependent role of Grb7 in HER2+ve and triple-negative breast cancer cell lines. Breast Cancer Res Treat 143: 593-603, 2014.
23. Peng Y, Zhong Y and Li G: Tubeimoside-1 suppresses breast cancer metastasis through downregulation of CXCR4 chemokine receptor expression. BMB Rep 49: 502-507, 2016.

24. Lian X, Jiao Y, Yang Y, Wang Z, Xuan Q, Liu H, Lu S, Wang Z, Liu Y, Li S, et al: CrkL regulates SDF-1-induced breast cancer biology through balancing Erk1/2 and PI3K/Akt pathways. Med Oncol 32: 411, 2015

25. Lin J, Ma T, Jiang X, Ge Z, Ding W, Wu Y, Jiang G, Feng J, Cui G and Tan Y: JWA regulates human esophageal squamous cell carcinoma and human esophageal cells through different mitogen-activated protein kinase signaling pathways. Exp Ther Med 7: 1767-1771, 2014

26. Lu J, Tang Y, Cheng Y, Zhang G, Yip A, Martinka M, Dong Z, Zhou $\mathrm{J}$ and Li G: ING4 regulates JWA in angiogenesis and their prognostic value in melanoma patients. Br J Cancer 109: 2842-2852, 2013

27. Qian J, Zhu W, Wang K, Ma L, Xu J, Xu T, Røe OD, Li A, Zhou J and Shu Y: JWA loss promotes cell migration and cytoskeletal rearrangement by affecting HER 2 expression and identifies a high-risk subgroup of HER 2-positive gastric carcinoma patients. Oncotarget 7: 36865-36884, 2016.

28. Chen X, Feng J, Ge Z, Chen H, Ding W, Zhu W, Tang X, Chen Y, Tan Y and Ma T: Effects of the JWA gene in the regulation of human breast cancer cells. Mol Med Rep 11: 3848-3853, 2015. International (CC BY-NC-ND 4.0) License. 